\title{
Quantitative and qualitative study of endogenous and exogenous growth regulators in eggplant (Solanum melongena) microspore cultures
}

\author{
Antonio Calabuig-Serna ${ }^{1} \cdot$ Carolina Camacho-Fernández $^{1} \cdot$ Ricardo Mir $^{1} \cdot$ Rosa Porcel $^{1} \cdot$ Esther Carrera $^{2}$. \\ Isabel López-Díaz ${ }^{2}$. Jose M. Seguí-Simarro ${ }^{1}$ (1)
}

Received: 22 June 2021 / Accepted: 18 November 2021 / Published online: 26 November 2021

(c) The Author(s) 2021

\begin{abstract}
In eggplant microspore embryogenesis, embryos are produced and then transformed into undifferentiated calli, instead of developing as true embryos. This is the main current bottleneck that precludes this process from being efficient. In this work we aimed to shed light on the factors involved in the successful in vitro development of eggplant haploid embryos by evaluating the role of growth regulators (GRs) in this process. We analyzed the endogenous levels of different GRs, including auxins, cytokinins and gibberelins, as well as salicylic, jasmonic and abscisic acid, in microspores and microspore-derived embryos at different culture stages. We also analyzed the same GR profiles in leaf and anther wall tissues of different eggplant backgrounds. Finally, we assessed the application of different GR combinations to the culture medium. Our results showed that in eggplant there are no genotype-specific endogenous GR profiles that can be associated to a high embryogenic response. Instead, the embryogenic response seems related to different GR accumulation patterns during in vitro culture. The changes observed in the endogenous levels of salicylic and abscisic acid were not related to the embryo transition. There were, however, changes in the levels of indole acetic acid and dihydrozeatin. The best GR combination to promote callus production was $0.5 \mathrm{mg} / \mathrm{L} 1$-naphthaleneacetic acid (NAA) and $0.5 \mathrm{mg} / \mathrm{L}$ 6-benzylaminopurine (BAP). A $20 \%$ reduction of NAA and BAP reduced embryo production but produced structures more anatomically similar to embryos. These results shed light on the role of GRs during the development of microspore-derived embryos in eggplant microspore cultures.
\end{abstract}

Keywords Androgenesis $\cdot$ Hormones $\cdot$ Microspore culture $\cdot$ Microspore embryogenesis $\cdot$ Solanum melongena

\section{Introduction}

Microspore embryogenesis is an experimental pathway whereby some microspores are deviated from their original gametophytic fate and induced in vitro to form new haploid

Communicated by Stefaan Werbrouck.

Antonio Calabuig-Serna and Carolina Camacho-Fernández have contributed equally to the work.

Jose M. Seguí-Simarro

seguisim@btc.upv.es

1 Cell Biology Group - COMAV Institute, Universitat Politècnica de València, 46022 Valencia, Spain

2 Instituto de Biología Molecular y Celular de Plantas (CSIC- Universitat Politècnica de València), 46022 Valencia, Spain or doubled haploid (DH) microspore-derived embryos (MDEs). Other microspores follow a sporophytic-like pathway (Satpute et al. 2005) and many others just arrest and die. Microspore embryogenesis is a very convenient tool for plant breeding purposes (Weyen 2021), as it allows for a fast production of fully homozygous plants. Microspore embryogenesis is also useful for fundamental research $(\mathrm{Li}$ et al. 2014; Jouannic et al. 2001), as microspore cultures are convenient haploid platforms for genetic studies and genetic transformation, and model systems to study in vitro embryogenesis. Microspore cultures have the potential to yield high quantities of DHs, since a single anther contains thousands of potentially embryogenic microspores. However, the low embryogenic response of some species still limits the use of this DH technology.

The efficiency of microspore cultures is hard to predict because there are many factors involved. They can be grouped in three main categories (reviewed in Seguí-Simarro 
et al. 2011). The first relates to conditions of the donor plants, among which the genotype is the most important. The second relates to the stage of the microspores. For most species, the only developmental stages where microspores/ pollen are responsive to the induction treatments are the vacuolated microspore and the young pollen grain (SeguíSimarro et al. 2021). The third category involves the stress treatments and culture conditions applied. The type and concentration of plant growth regulators (GRs) is a critical parameter. GRs play a relevant role in plant physiology and development, including embryogenesis, and are essential to promote in vitro morphogenic processes. In line with this, GRs are in general needed for successful microspore embryogenesis. However, species with higher endogenous GR levels require less application of exogenous analogs (Żur et al. 2015). In Brassica napus, low levels of endogenous GRs may alter the transition from globular to heart-shaped MDEs (Ramesar-Fortner and Yeung 2006). In barley, abscisic acid (ABA) seems beneficial to increase viability by preventing cell death and the microspore-to-pollen transition (van Bergen et al. 1999). At the GR level, there are differences between zygotic and MDEs. For example, in B. napus, indole acetic acid (IAA) and ABA levels are lower in MDEs than in zygotic embryos (Hays et al. 2001).

In eggplant (Solanum melongena), the most broadly used approach to produce DHs is anther culture (Salas et al. 2011, 2012; Calabuig-Serna et al. 2021). Advances have been made in microspore cultures, but this technique is not yet well developed (reviewed in Seguí-Simarro 2016). Miyoshi (1996) described the first protocol for embryogenesis induction, callus production and regeneration of DHs from isolated eggplant microspores, establishing the basis of the inductive conditions and the composition of the culture medium. Since then, several medium modifications have been tested to improve the efficiency of the process and to stimulate MDE progression, with different results. The addition of polyethylene glycol improved callus induction and proliferation (Corral-Martínez and Seguí-Simarro 2012), while the addition of epibrassinolide improved callus induction but had a negative effect on callus proliferation (Corral-Martínez and Seguí-Simarro 2014). Higher concentrations of Nitsch and Nitsch (NLN) salts and sucrose were found beneficial, but only for some genotypes (Rivas-Sendra et al. 2020). The addition of ABA prevented disorganization at the beginning of the culture, but could not prevent the transformation of globular MDEs into calli (Corral-Martínez and Seguí-Simarro 2014). This is currently the main bottleneck of the protocol. Production of microspore-derived calli instead of embryos has been described in different species as dependent on many factors, including the genotype, the induction temperature (Parra-Vega et al. 2013), and the levels and relative proportions of GRs. Aside of the works mentioned above, nothing else is known about the role of
GRs in eggplant microspore embryogenesis. Thus, there is still much room for improvement of the method.

In this work, we aimed to study the endogenous GR levels of eggplant cultured microspores in order to find ways to modulate the response and, principally, to overcome the blockage of the transition from globular to heart-shaped MDEs. Then, we tried to modulate the auxin:cytokinin ratio by modifying the levels and proportions of 1-naphthaleneacetic acid (NAA) and 6-benzylaminopurine (BAP), the auxin and cytokinin used in the protocol. Although these are synthetic GRs, they are known to alter the endogenous GR levels when applied exogenously, depending on the species, tissues and morphogenic processes studied. They are also known to directly affect the hormonal responses. There are abundant examples of application of NAA, and exogenous auxins in general, to increase the endogenous levels of IAA, for example, in rooting hypocotyl cuttings of soybean (Liu et al. 1996), and in rooting single-node cuttings of hybrid aspen (Yan et al. 2017). It was also found that NAA promoted a remarkable increase of both free and conjugated forms of IAA during somatic embryogenesis in Coffea canephora (Ayil-Gutiérrez et al. 2013). A similar result was found with the application of 2,4-dichlorophenoxyacetic acid (2,4-D) for somatic embryogenesis in Abies alba (Vondráková et al. 2011). Our results shed light on the role of these exogenously applied GRs in eggplant microspore embryogenesis.

\section{Materials and methods}

\section{Plant material}

We used plants of the eggplant DH36 line (Rivas-Sendra et al. 2017) as donors of microspores. For the analysis of GR profiles in different plant tissues we used DH36 and two eggplant F1 hybrids, identified as E1 and E2 for this study, since they are proprietary material protected under a confidentiality agreement. Plants of each line were grown in $30 \mathrm{~cm}$ pots at the greenhouse under controlled temperature $\left(25^{\circ} \mathrm{C}\right)$ and natural light.

\section{Isolated microspore culture}

Isolated microspore culture was performed according to Calabuig-Serna et al. (2021). Briefly, anthers containing mostly vacuolated microspores were dissected from the bud, surface disinfected with $70 \%$ ethanol for $30 \mathrm{~s}$ and with $4 \mathrm{~g} / \mathrm{L}$ sodium hypochlorite for $5 \mathrm{~min}$, and rinsed three times in sterile $\mathrm{dH}_{2} \mathrm{O}$. Anthers were crushed in a small beaker with sterile $\mathrm{dH}_{2} \mathrm{O}$ using a syringe piston and their locular content was isolated by filtration through a $41 \mu \mathrm{m}$ nylon mesh (Millipore). Then, three centrifugation steps were performed at 
$100 \times g$ for 4 min each. The initial microspore density was calculated with a Neubauer Improved chamber (CamachoFernández et al. 2018). Then, the microspore suspension was diluted with the necessary volume of induction medium (sterile $\mathrm{dH}_{2} \mathrm{O}$ ) to reach a plating density of 200,000 microspores/mL (Rivas-Sendra et al. 2020). The microspore suspension was collected with a pipette and distributed in sterile plastic culture dishes ( $6 \mathrm{~cm}$ diameter), pouring $3 \mathrm{~mL}$ in each plate. Plated microspores were incubated at $35^{\circ} \mathrm{C}$ in the dark for 3 days to induce embryogenesis. Then, microspores were collected by centrifugation, resuspended at the same density in culture medium, and incubated continuously at $25^{\circ} \mathrm{C}$ in the dark. The basal culture medium used for eggplant microspore culture included NLN salts and vitamins, $2 \%$ sucrose, $0.5 \mathrm{mg} / \mathrm{L}$ NAA and $0.5 \mathrm{mg} / \mathrm{L}$ BAP. All the tissue culture chemicals, including media and hormones, were purchased from Duchefa, Netherlands.

\section{Modifications of GR concentration}

The above mentioned GR concentrations were used as references for further modifications, and expressed as 100\% GR. Further modifications consisted in changes in the concentration of one or both GRs, and were expressed as percentages too. For example, an experiment with $20 \%$ GR meant that one fifth (20\%) of the initial (100\%) NAA and BAP concentration was used. When different modifications were applied to NAA and to BAP, they were specified using the same terminology. For the second batch of experiments, we increased the sucrose and NLN salts concentration to $2 \times$, since it previously showed a clearly positive effect in DH36, as opposed to other eggplant backgrounds (Rivas-Sendra et al. 2020). Microspore cultures performed under different conditions were repeated at least three times. The changes of GR concentrations were done at specific culture stages: day 3 (once the inductive treatment is finished and microspores are moved to continuous $25{ }^{\circ} \mathrm{C}$ incubation), day 7 (when microspores start cell proliferation as MDEs), and day 12 (when globular MDEs begin to form in the culture).

As an estimation of the effect of each treatment, microspore-derived structures were observed and counted after approximately one month of culture. Counts were expressed as number of structures per $\mathrm{mL}$ of culture medium, normalized to the value of the experiment considered as reference (100\% GR for the first batch of experiments and 20\% GR for the second batch). Counting of the microspore-derived structures and analysis of their treatment-associated morphological differences were performed by direct observation and imaging of culture dishes with stereo and inverted microscopes. An ANOVA test $(\mathrm{p} \leq 0.05)$ was performed to assess global statistically significant differences. A Fisher's least significant difference (LSD) test for multiple comparisons was performed to group the effect of the treatments, considering the differences as significant when the $\mathrm{p}$-value was $<0.05$. Planned orthogonal contrasts were performed to obtain additional information about comparisons between sample groups. For the second batch of experiments, statistically significant differences among experiments were determined using the Kruskal-Wallis test.

\section{Quantification of plant GRs}

Microspore cultures were collected at different stages by centrifugation, separating the pellet (only microspores and MDEs) from the supernatant (the conditioned medium where the microspores and MDEs have grown). As a control for the conditioned medium, we also prepared and analyzed for each stage a batch of fresh medium $\left(\mathrm{dH}_{2} \mathrm{O}\right.$ for the induction stages from day 0 to 3 , and culture medium for the rest of stages). The culture media $(4.5 \mathrm{~mL})$ were lyophilized to produce a dry residue to be processed. Plant tissues were directly excised from plants. Samples were taken from leaves of the same size and position (shoot apex) in the plant. Anther samples were taken from flowers of the main inflorescences of the plants, at the stage when anthers contain mostly vacuolated microspores and young pollen (Salas et al. 2012). In both cases, samples were taken from plants of the same age. About 50-200 mg (fresh weight) of each sample (plant tissues, microspores/MDEs and lyophilized culture media) were thoroughly ground (except for lyophilized media), suspended in $80 \%$ methanol- $1 \%$ acetic acid containing internal standards, and mixed by shaking during $1 \mathrm{~h}$ at $4{ }^{\circ} \mathrm{C}$. The extract was kept a $-20^{\circ} \mathrm{C}$ overnight and then centrifuged. The supernatant was dried in a vacuum evaporator. The dry residue was dissolved in $1 \%$ acetic acid and passed through a reverse phase column (HLB Oasis $30 \mathrm{mg}$, Waters), as described in Seo et al. (2011). The extracts were additionally passed through an Oasis MCX (cationic exchange) and eluted with $60 \%$ methanol- $5 \% \mathrm{NH}_{4} \mathrm{OH}$ to obtain the basic fraction containing cytokinins. To recover the acid fraction, the MCX cartridge was eluted with methanol. The final residues were dried and dissolved in 5\% acetonitrile-1\% acetic acid and the GRs were separated by ultra high perfomance liquid chromatography (UHPLC) with a reverse Accucore C18 column ( $2.6 \mu \mathrm{m}, 100 \mathrm{~mm}$ length; ThermoFisher Scientific) with an acetonitrile gradient containing $0.05 \%$ acetic acid, at $400 \mu \mathrm{L} / \mathrm{min}$. For cytokinins, the acetonitrile gradient was $2-25 \%$ over 13 min. For gibberellic acids (GAs), IAA, ABA, jasmonic acid (JA) and salicylic acid (SA) the gradient was $2-55 \%$ acetonitrile over $21 \mathrm{~min}$. The GRs were analyzed with a Q-Exactive mass spectrometer (Orbitrap detector; ThermoFisher Scientific) by targeted Selected Ion Monitoring (tSIM; capillary temperature $300{ }^{\circ} \mathrm{C}$, S-lens RF level 70, resolution 70.000) and electrospray ionization (spray voltage $3.0 \mathrm{kV}$, heater temperature $150{ }^{\circ} \mathrm{C}$, sheath gas flow rate $40 \mu \mathrm{L} / \mathrm{min}$, auxiliary gas flow rate $10 \mu \mathrm{L} / \mathrm{min}$ ) 
in negative mode for acidic GRs or positive mode for cytokinins. The concentrations of GRs in the extracts were determined using embedded calibration curves and the Xcalibur 4.0 and TraceFinder 4.1 SP1 programs. The internal standards for quantification of each of the different plant GRs were the deuterium-labelled GRs, except for JA, for which the compound dhJA was used (purchased from OlChemim Ltd, Olomouc, Czech Republic). Statistical analysis was performed using SPSS 16.0 statistical program (SPSS Inc., Chicago, IL, USA). Data were subjected to analysis of variance (ANOVA) with plant genotype (G), tissue (T) and their interactions as sources of variation. The Duncan's test was used to find out differences between groups $(\mathrm{p} \leq 0.05)$.

\section{Results}

\section{Induction of eggplant microspore embryogenesis}

Typically, eggplant microspore cultures started with the isolation and plating of microspores at the right stage of development (vacuolated microspores and young pollen grains, Fig. 1A). After three days of heat shock-based inductive treatment (Fig. 1B), some microspores enlarged, whereas others remained arrested. In 7-day-old cultures, the first clearly embryogenic structures could be identified (arrowhead in Fig. 1C). Between 2 and 3 weeks after culture initiation, globular MDEs (Fig. 1D) transformed into undifferentiated, proliferative calli. Individualization and culture of calli in solid medium produced organogenic nodules from which shoots emerged (Fig. 1E). Shoots were excised and transferred to rooting medium, where they rooted and grew to become full in vitro plantlets (Fig. 1F). Three-four months after culture initiation, DH plantlets were ready for transplantation to pots and acclimatization to ex vitro conditions (Fig. 1G).

\section{Analysis of GR profiles during eggplant microspore embryogenesis}

We analyzed the endogenous levels of different GRs, including auxin (IAA), cytokinins such as trans-zeatin (tZ), dihydrozeatin (DHZ) and isopentenyl adenine (iP), gibberelins $\left(\mathrm{GA}_{1}\right.$ and $\left.\mathrm{GA}_{4}\right), \mathrm{ABA}, \mathrm{SA}$ and JA. Among them, $\mathrm{tZ}$ was undetectable, most likely because its levels were always below the system resolution. The levels of gibberelins and JA were very low and inconsistent. Thus, we decided to discard them. iP presented relatively constant values, ranging around 5-7 ng/g and with no relevant changes among stages (data not shown). However, notable differences were found
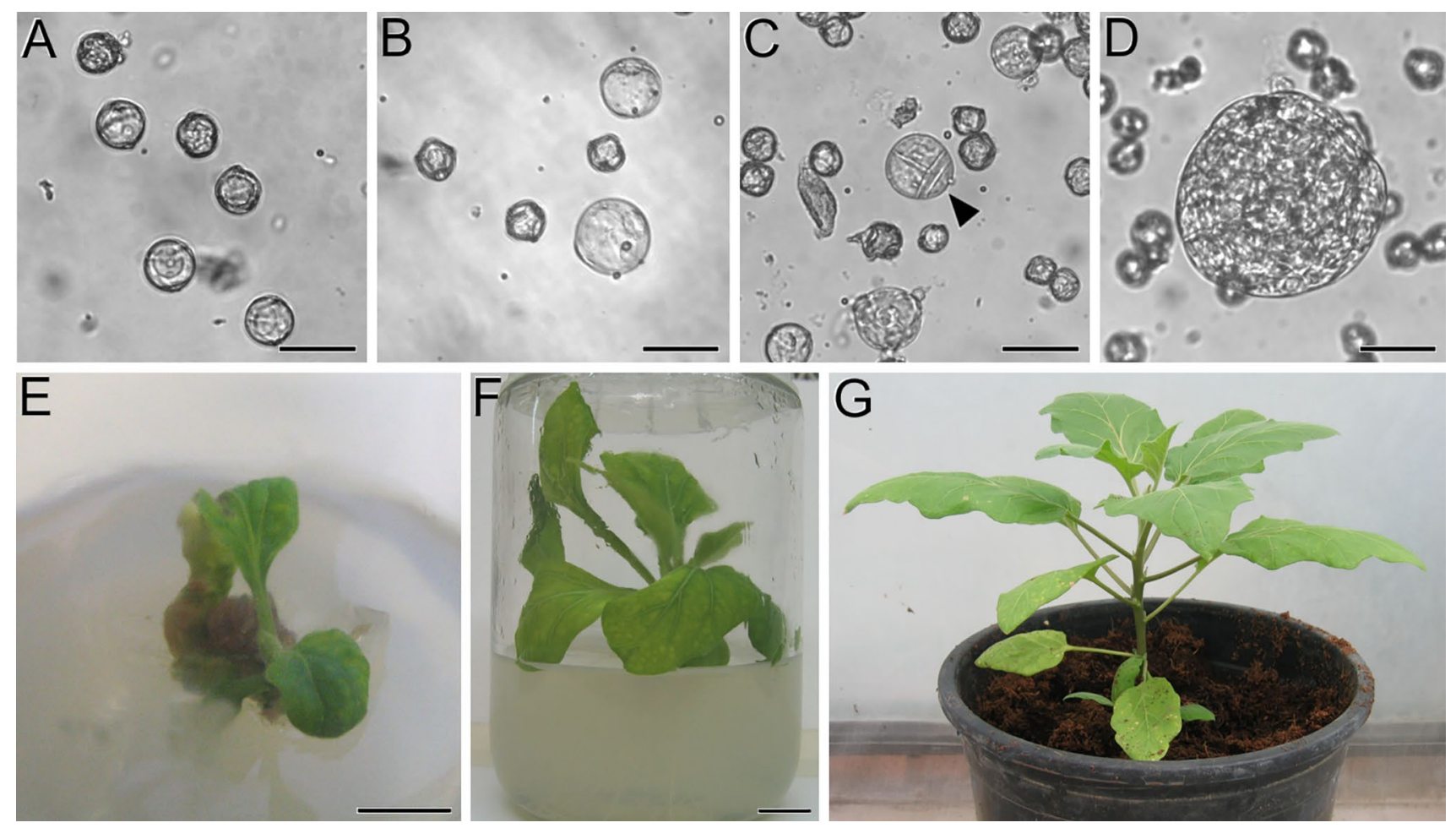

Fig. 1 Eggplant microspore culture. A Culture at day 0 (freshly isolated microspores). B Three-day-old culture, after the inductive treatment. Note the enlarged microspores, sensitive to the inductive treatment. C Culture at day 7. Arrowhead points to a dividing micro- spore-derived structures. D Globular MDE in 13 day-old culture. E Organogenic callus with a shoot. F: Rooted, full in vitro plantlet. G: Acclimated eggplant DH plant. Bars in A-D: $20 \mu \mathrm{m} ; \mathbf{E}, \mathbf{F}: 1 \mathrm{~cm}$ 
for IAA, ABA, DHZ and SA. The endogenous levels of IAA (Fig. 2A) in cultured microspores showed a peak at day 2 $(\sim 8.3 \mathrm{ng} / \mathrm{g})$, still during the induction treatment. Then, the levels progressively decreased to $\sim 3 \mathrm{ng} / \mathrm{g}$ at day 7 , and then they remained relatively stable. The conditioned medium followed the same pattern, but with levels remarkably higher. Indeed, the day-2 IAA levels peaked to $\sim 180 \mathrm{ng} / \mathrm{g}$ and then dropped down and stabilized at around 45-60 ng/g until day 21 , when they decreased to $\sim 5 \mathrm{ng} / \mathrm{g}$. The levels of IAA in fresh medium (considered as background) were always between $0.3-0.9 \mathrm{ng} / \mathrm{g}$. The endogenous ABA levels (Fig. 2B) were $\sim 5 \mathrm{ng} / \mathrm{g}$ in freshly isolated microspores, decreased to $\sim 1 \mathrm{ng} / \mathrm{g}$ during the induction treatment, and then increased to reach the initial levels at day 21 . The levels of conditioned medium were in general low but not negligible, indicating a low ABA secretion, except for microspores at days 14-21, where the increase observed in their endogenous levels correlated with a dramatic increase in the conditioned medium, indicative of massive ABA secretion. The levels of fresh medium were always negligible, below $0.1 \mathrm{ng} / \mathrm{g}$. The endogenous levels of DHZ (Fig. 2C) were in general very low in microspores, revolving around $0.8-0.9 \mathrm{ng} / \mathrm{g}$ during the induction treatment and until day 4 , and then decreasing to nearly zero. The levels of both fresh and conditioned medium were negligible, indicating no DHZ secretion. The SA levels (Fig. 2D) were in general low except for days 7-14, when the medium levels increased notably, suggesting a massive SA secretion at these stages.

In summary, the endogenous IAA levels increased during the first culture stages, whereas the cytokinin levels remained unchanged or decreased, as for DHZ, which could possible produce a misbalance of the auxin:cytokinin ratio. There were significant differences between fresh media and conditioned media, indicating that cultured microspores
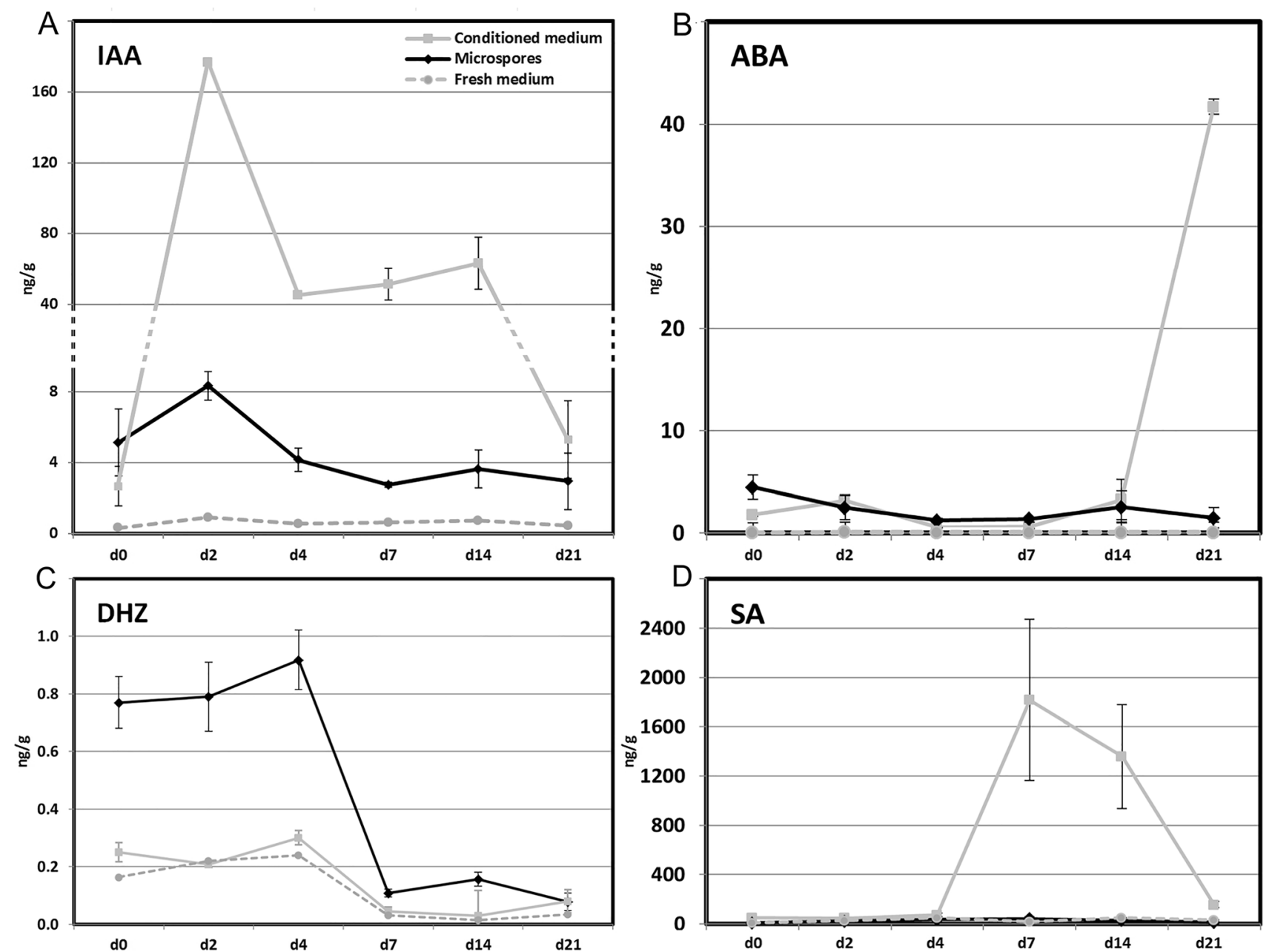

Fig. 2 Levels of GRs in DH36 cultured microspores (black filled diamond), fresh (gray filled circle) and conditioned medium (gray filled square) at different culture stages: day 0 (freshly isolated microspores, before induction), day 2 (during the heat shock and starvation induc-

tive treatment), day 4 (after the inductive treatment, and 1 (d7), 2 (d14) and 3 weeks after culture initiation (d21). A IAA, B ABA, C DHZ, D SA. Results are expressed in ng of GR per $g$ of sample dry weight $(\mathrm{ng} / \mathrm{g}) \pm$ s.e. 
secreted GRs to the medium, thereby conditioning it for further culture stages.

\section{Analysis of GR profiles in tissues of different eggplant backgrounds}

Next, we wanted to check out whether the GR profiles of the DH36 genotype were different from other, low responding backgrounds. For this, we compared the endogenous GR levels of different DH36 tissues (microspores, anther walls excluding microspores, and leaves) with those of E1 and E2, known to have a markedly lower embryogenic response ( $\sim 200$ calli $/ \mathrm{mL}$ for $\mathrm{DH} 36$ vs $\sim 20$ calli $/ \mathrm{mL}$ for $\mathrm{E} 1$ and $\mathrm{E} 2$, as reported in Rivas-Sendra et al. 2020). We focused on the GRs that showed relevant changes during microspore embryogenesis, namely IAA, ABA, DHZ and SA. The DHZ and SA levels were not significantly different among genotypes (data not shown). The levels of IAA were similar in leaves of the three genotypes (Fig. 3A). In anthers, the IAA levels were higher than in leaves, but also similar among genotypes. In microspores, the IAA levels were lower than in leaves and anther walls, and different among genotypes. E1 presented levels significantly higher than DH36 and E2, whose levels were not different (Fig. 3A). For ABA, the

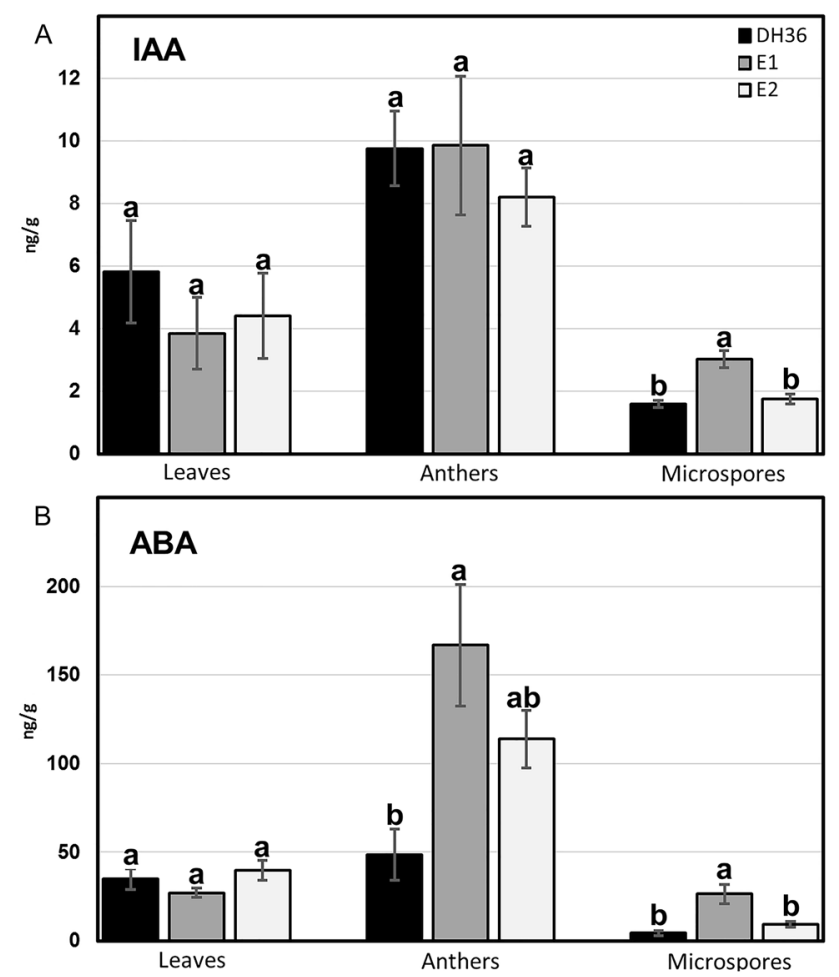

Fig. 3 Endogenous levels of IAA (A) and ABA (B) in DH36, E1 and E2 leaves, microspores and anther walls (without microspores). Results are expressed in ng of GR per $\mathrm{g}$ of sample dry weight $(\mathrm{ng} / \mathrm{g}) \pm$ s.e. levels were similar in leaves of the three genotypes (Fig. 3B). In anthers, the ABA levels were in general higher than in leaves and presented clear differences among genotypes. In microspores, the ABA levels were in general lower than in leaves and anther walls, and differed among genotypes. E1 presented levels significantly higher than DH36 and E2, whose levels were not different (Fig. 3B). As seen, specific GR profiles for DH36, different from the two low responding genotypes E1 and E2, could not be identified in any tissue. For DHZ and SA the levels of the three genotypes were not different, whereas for IAA and ABA, the levels of DH36 were similar to one of the low responding genotypes (E2) and lower than the other (E1). In conclusion, the GR levels of the DH36 tissues and of microspores in particular did not appear related to the high embryogenic competence of this genotype.

\section{Response to changes in GRs}

Our previous results showed that reducing the concentration of GRs could increase the induction rates (Corral-Martínez and Seguí-Simarro 2014). Thus, we reduced the initial GR concentration ( $100 \%$, normalized to 1 ) to $20 \%, 10 \%$ or $0 \%$ at 7 and 12 days from the isolation of DH36 microspores, according to the design shown in Fig. 4. All the GR combinations induced microspore divisions and callus growth, except for the $0 \%$ GR (no GRs at any stage), which produced no calli. Statistical analysis of the effect of treatments in callus production revealed that the number of total calli was significantly affected by the treatments (Table 1), although none of the GR reductions tested increased the overall response in terms of total callus production. A detailed analysis of the data by means of planned contrasts (Table 1) showed that, in general, a reduction in GR concentration did not significantly improve callus formation. When GR levels were reduced either at days 7 or 12, an initial concentration of $100 \%$ GR produced significantly more calli than $20 \%$ GR. In addition, a partial reduction from 100 to $20 \%$ GR or from 20 to $10 \%$ GR produced more calli than a full reduction (0\%). However, the days when the reduction was applied appeared irrelevant. In summary, the results indicated that, in terms of callus production, the best GR combination was still $100 \%$, without reduction.

Our parallel morphological observations provided alternative data. Cultures starting with $100 \%$ GR at day 3 (Fig. 5A-C) produced structures with a more disorganized, fluffy morphology than those starting with $20 \%$ GR (Fig. 5D-F), irrespective of the GR reduction applied later on. Indeed, when the GR concentration was reduced from 20 to $0 \%$, either at days 7 (Fig. 5E) or 12 (Fig. 5F), the morphology of the structures formed was more similar to MDEs, with roots consistently appearing in many of the structures observed, although still with severe malformations in the 


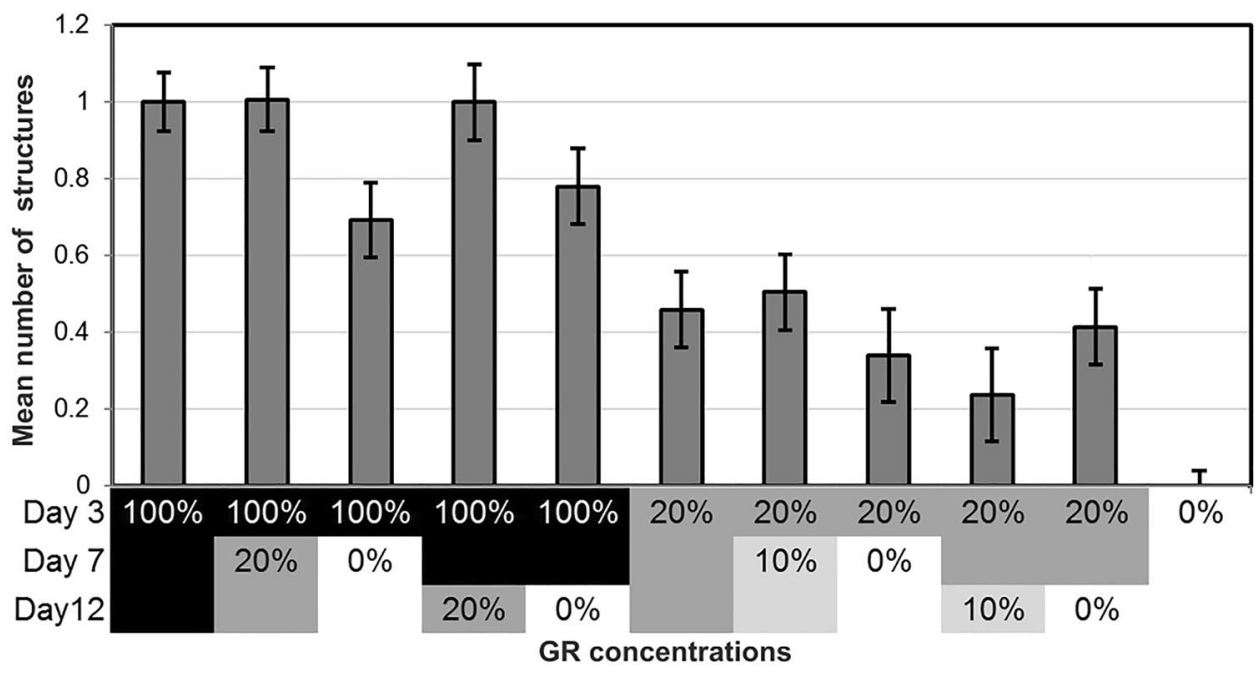

Fig. 4 Mean \pm s.e. of the number of microspore-derived structures produced using different GR concentrations. The number of microspore-derived structures produced with the concentration of reference (100\% the initial GR concentration, $0.5 \mathrm{mg} / \mathrm{L}$ NAA and $0.5 \mathrm{mg} / \mathrm{L}$ BAP) is normalized to 1 . The number of microspore-derived struc- tures produced using the rest of concentrations are transformed accordingly. The percentages of the $\mathrm{X}$-axis indicate the percentage of GRs used with respect to the reference (100\% GR). When performed, changes in GR concentrations at days 3, 7 and/or 12 are indicated with different color codes
Table 1 ANOVA and planned contrasts of the effect of reducing GRs in the microspore culture medium, expressed as total callus production

\begin{tabular}{lrrll}
\hline Source & DF & \multicolumn{1}{l}{ SS } & F & $p$ \\
\hline Total & 32 & $189,794.06$ & \multicolumn{1}{l}{-} & - \\
Treatments & 10 & $177,473.39$ & 31.69 & $<0.001^{*}$ \\
1. Initial GR concentration (100\% vs 20\%) when reduced & 1 & $106,400.17$ & 189.99 & $<0.001^{*}$ \\
2. Partial vs. total GR reduction & 1 & $14,113.50$ & 25.20 & $<0.001^{*}$ \\
3. Reduction at day 7 vs day 12 & 1 & 2053.50 & 3.67 & 0.069 \\
4. Reduction vs no reduction & 1 & 418.13 & 0.75 & 0.397 \\
5. 100\% vs. 20\% GR when not reduced & 1 & $21,961.50$ & 39.21 & $<0.001^{*}$ \\
6. 100\% GR vs 20\% GR & 1 & $128,184.03$ & 228.89 & $<0.001^{*}$ \\
7. 100\% GR vs 100\% GR reduced after either 7 or 12 days & 1 & 570.42 & 1.02 & 0.324 \\
8. 20\% GR vs. 20\% GR reduced after either 7 or 12 days & 1 & 25.35 & 0.05 & 0.833 \\
Error & 22 & $12,320.67$ & - & \\
\hline
\end{tabular}

$D F$ degrees of freedom, $S S$ sum of squares, $F$ F-value, $p p$-value

Asterisks represent significant differences between the groups of each contrast shoot apex (Fig. 5F). These observations suggested that the presence of GRs in the culture medium is essential to promote growth at least during the first days after the inductive treatment, and that a reduction of GRs to $20 \%$ might help to form MDEs instead of calli. In addition, the massive secretion of IAA to the medium might be conditioning it for the next stages of MDE progression, thereby generating a misbalanced auxin:cytokinin ratio, which could possibly be compensated by increasing the levels of exogenously applied cytokinin. In other words, different ratios of exogenous GRs during MDE progression might be necessary for an optimized development.

To check these hypotheses out, we designed a second batch of GR combinations taking $20 \%$ GR as the reference (normalized to 1), using reduced percentages of GRs and changing these percentages and the auxin:cytokinin ratio at defined stages, according to the design shown in Fig. 6. Among all the combinations tested, only the continuous culture with $20 \%$ GRs performed significantly better than the rest. Other GR combinations including changes at days 3, 7 and/or 12 were not as effective. Altering the auxin:cytokinin ratio at different stages did not have the expected results, always performing below $20 \%$ GR. From a qualitative point of view, the morphology of the structures produced with the different GR combinations was similar to those shown in Fig. 5. As in the previous experiment, a reduction of the GR levels at days 3, 7 and/or 12 produced much less structures, but they were more similar 

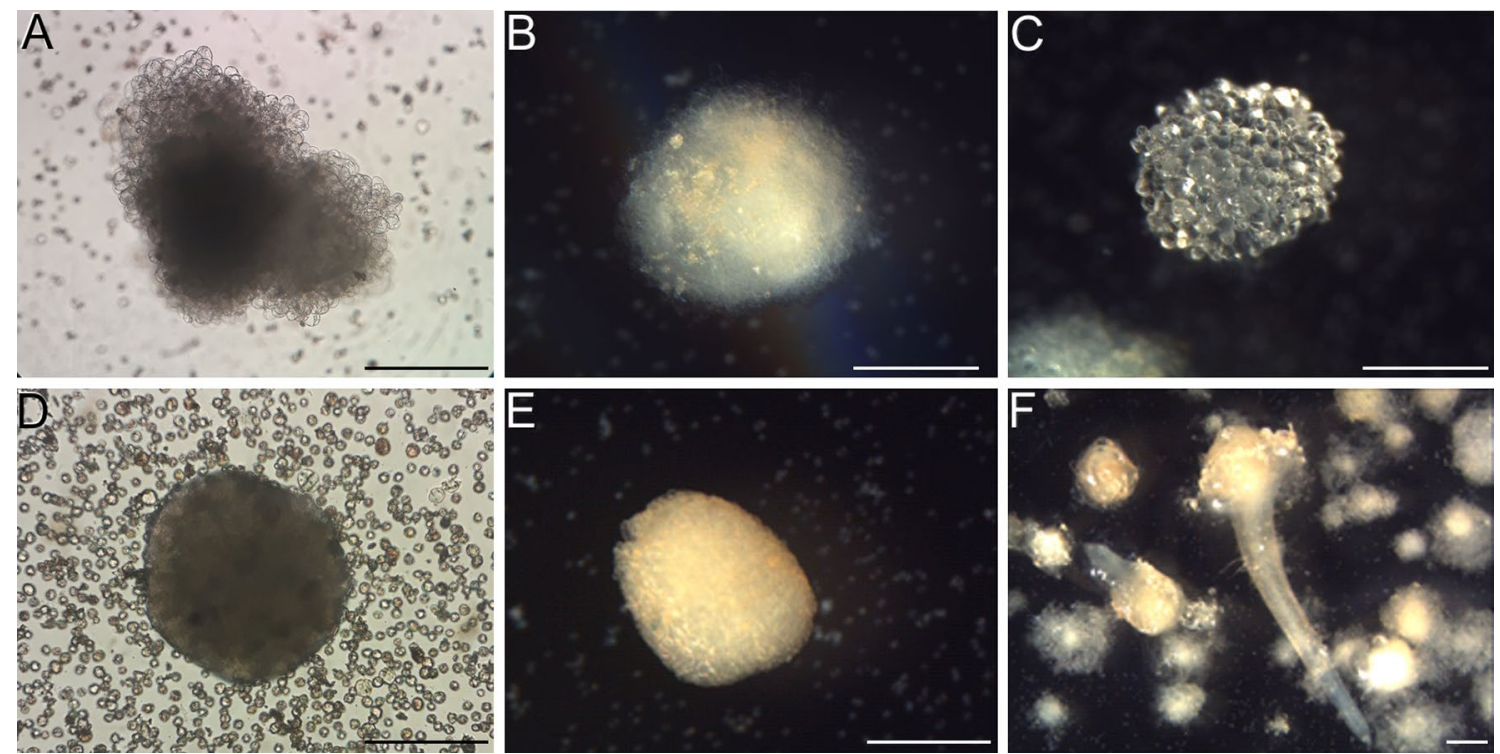

Fig. 5 Microspore-derived structures observed in DH36 microspore cultures with different concentrations of GRs. A $100 \%$ GR continuously. B $100 \%$ GR reduced to $20 \%$ at day 12 . C $100 \%$ GR reduced to $0 \%$ at day 7. D $20 \%$ GR continuously. E $20 \%$ GR reduced to $0 \%$ at day 7 . F $20 \%$ GR reduced to $0 \%$ at day 12 . Bars: $500 \mu \mathrm{m}$

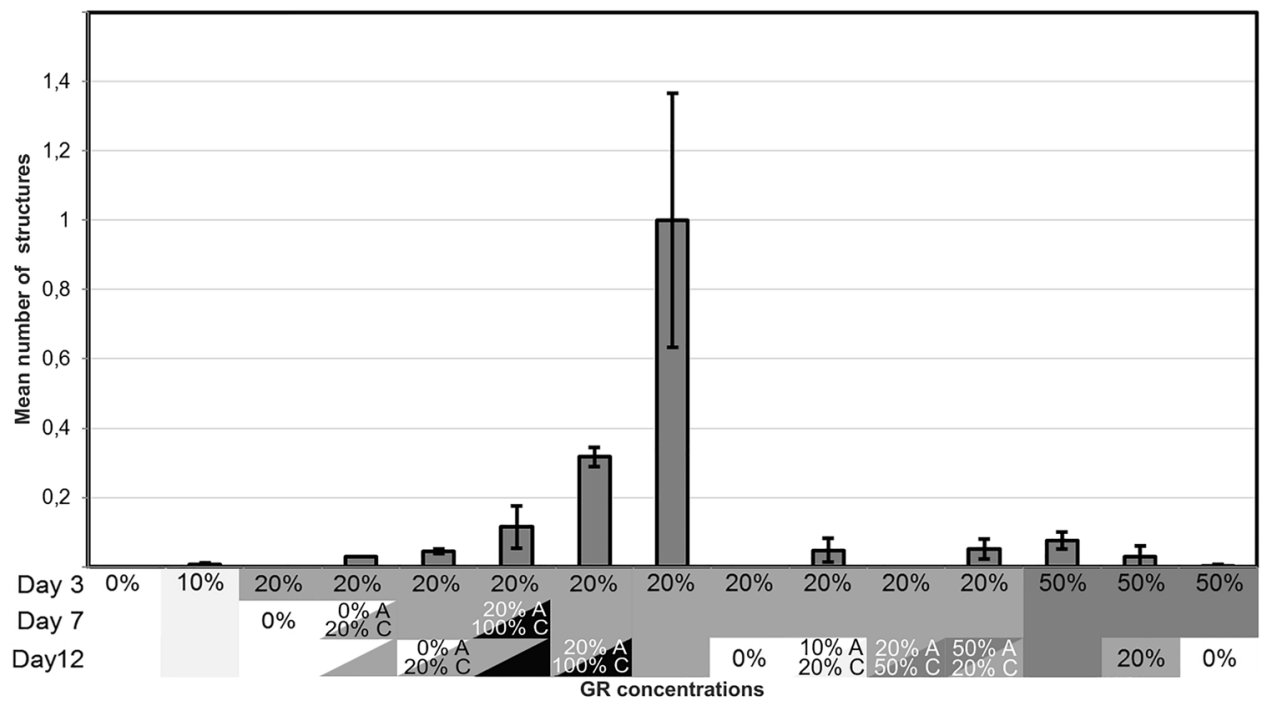

Fig. 6 Mean \pm s.e. of the number of microspore-derived structures produced using different GR concentrations. The number of microspore-derived structures produced with the concentration of reference (20\% the initial GR concentration, $0.1 \mathrm{mg} / \mathrm{L}$ NAA and $0.1 \mathrm{mg} / \mathrm{L}$ $\mathrm{BAP}$ ) is normalized to 1 . The number of microspore-derived struc-

to MDEs. Nevertheless, no bipolar, functionally and anatomically normal MDEs were observed. In summary, the continuous use of $100 \%$ GR $(0.5 \mathrm{mg} / \mathrm{L} \mathrm{NAA}$ and $0.5 \mathrm{mg} / \mathrm{L}$ BAP) was confirmed as the best condition to induce eggplant microspores to embryogenesis, but not to promote the growth of MDEs. For the latter, the continuous use of $20 \%$ GR was better. tures produced using the rest of concentrations are transformed accordingly. The percentages of the X-axis indicate the percentage of GRs used with respect to the reference (20\% GR). When performed, changes in the concentrations of auxin (A), cytokinin (C) or both at days 3, 7 and/or 12 are indicated with different color codes

\section{Discussion}

\section{The changes in SA and ABA levels do not appear related to MDE transition}

The analysis of the endogenous GRs levels during 
microspore embryogenesis revealed that there are two main stages in terms of GR profiles, up to day four and after day four. Up to day four IAA and DHZ showed the highest levels and then, they dropped down and stabilized. Similar IAA and cytokinin profiles have been observed in equivalent stages of somatic embryogenesis in other species (Pescador et al. 2012). They were attributed to the signaling needed to initiate the morphogenic process. In the latest stages, all GRs kept similarly low endogenous levels, but with very different patterns of secretion, whose biological significance is open to question. There was a massive secretion of SA between days 7 and 14, and of ABA at day 21, where SA levels dropped down. SA has been related to a variety of processes including defensive responses against pathogens or abiotic stresses, and in the regulation of many different physiological and biochemical processes during the plant life cycle (Rivas-San Vicente and Plasencia 2011). However, its role during embryogenesis has been restricted to seed germination. No evidence of a role during the transition from globular to heart-shaped embryos has been reported, which makes us suggest that the increase in the levels of SA secreted to the culture medium might be related to a response against some of the abiotic stresses concurring in the in vitro culture environment.

During microspore embryogenesis, ABA has been related to abiotic stress (Żur et al. 2015), whereas during zygotic embryogenesis, ABA is involved in the regulation of embryo development and seed maturation (desiccation, dormancy and inhibition of premature germination), coinciding with two peaks of ABA accumulation. The first peak is due to the synthesis of ABA in maternal tissues, and the second is produced by the embryo at late stages of seed maturation (Cheng et al. 2014). In our in vitro embryogenesis system, we did not observe any peak comparable to such first peak. In other in vitro embryogenesis systems like somatic embryogenesis in Acca sellowiana, such first peak was also absent (Pescador et al. 2012). In both cases, this is consistent with the maternal origin of this ABA peak. The peak we observed at day 21, when embryos are transforming into calli, could well be attributed to the deregulation of the embryogenic program. Indeed, in other in vitro embryogenesis systems, this peak is observed at late stages of maturation of the cotyledonary embryo (Pescador et al. 2012), whereas we observed it during the transition from globular MDE to callus. Since no cotyledonary MDEs but calli are produced from our eggplant structures at this stage, the peak should be attributable to callus growth or differentiation. In conclusion, our results do not point to a role for $\mathrm{ABA}$ and SA in MDE progression.

\section{Modification of exogenous NAA and/or BAP levels does not compensate for the change in the endogenous auxin:cytokinin ratio}

The two GRs expected to be more involved in MDE progression are auxin and cytokinins, since their exogenous application appears essential for eggplant microspore proliferation. After day four, the endogenous levels of IAA stabilized at levels comparable to those of freshly isolated microspores (day 0), whereas iP levels remained relatively constant and those of DHZ moved to levels much lower than those of day 0 . This change was paralleled by a massive release of IAA to the medium, whereas DHZ was not secreted at all. In short, microspore culture promoted a dramatic change in the auxin:cytokinin ratio inside microspores, but also in the culture medium, during the transition from globular to heart-shaped MDE. IAA and NAA are associated to callus proliferation and maintenance, as opposed to cytokinins, more related to organogenesis (Centeno et al. 1996). This is why we hypothesized that the high auxin:cytokinin ratios observed in eggplant microspore cultures could possibly be behind the inability of MDEs to successfully undergo such transition, and their subsequent transformation into undifferentiated calli.

In an attempt to overcome this bottleneck, we altered the GR concentrations at different time points and found that neither lower GR concentrations nor different auxin:cytokinin ratios had positive effects in the rate of MDE induction. In all cases, the MDE rate was not better than with continuous $100 \%$ GR. However, reduced GR levels showed an effect in the morphology of some of the structures observed, being more similar to conventional MDEs, and producing roots in many cases. This indicated that the endogenous auxin levels induced by the use of $100 \%$ GR were too high for a correct MDE development. This GR unbalance will most likely be affecting negatively to globular MDE progression and causing their transformation into calli.

\section{The embryogenic competence of eggplant genotypes seems not associated to specific endogenous GR levels}

Different species require different types and concentrations of GRs to induce microspore embryogenesis. Reasonably, the endogenous GR levels of each genotype might influence its embryogenic competence, as it occurs in other species and in vitro morphogenic processes. For example, different potato cultivars showed different response to in vitro adventitious shoot regeneration due to different endogenous levels of IAA (Pal et al. 2012), and in vitro suspension cells of different asparagus genotypes presented different contents of endogenous GRs (Campion et al. 1993). Unfortunately, no information on microspore culture systems is available. 
For this reason we compared the endogenous levels of DH36 microspores with those of other eggplant materials. We also analyzed other plant organs to evaluate whether such levels were specific for microspores or similar to other plant tissues. The levels we found were not suggestive of a special GR profile that could account for the differences observed in the embryogenic response of DH36 with respect to E1 and E2 (Rivas-Sendra et al. 2020), which discards genotype-specific GR profiles as responsible of the higher embryogenic competence of DH36. In turn, this suggests that the differences should be attributable to different GR accumulation patterns during in vitro microspore development.

\section{Concluding remarks}

In this work, we shed light on some of the reasons why eggplant MDEs do not develop well. For the most highly responding genotypes, it is not necessary to add GRs to the microspore culture medium. This is the case, for example, for some highly responding genotypes of tobacco (Touraev and Heberle-Bors 2003), maize (Vergne and Gaillard 2021) and B. napus (Corral-Martínez et al. 2021). On GR-free medium, these genotypes produce MDEs instead of calli, indicating that their endogenous GR levels are sufficient to promote the undisturbed differentiation of microspores into MDEs. In eggplant, however, the exogenous application of auxins and cytokinins appeared directly related to the amount of microspores that proliferate as MDEs, but inversely related to the quality of the MDEs obtained, unable to develop embryo-specific organs. Thus, the need for the exogenous application of GRs carries a compromise between quantity and quality difficult to resolve, at least with the currently available protocols. Nevertheless, quality should have priority over quantity, since quality is the main current bottleneck. Furthermore, any change in GR levels intended to optimize microspore development will have a different effect in different microspore-derived structures at different developmental stages, as mentioned above. This may be one of the reasons why some species, including eggplant, are still recalcitrant to microspore embryogenesis.

Author contributions ACS, CCF and RM performed eggplant microspore cultures. CCF, RP, EC and ILD performed GR analyses. CCF and JMSS designed the work. JMSS wrote the manuscript. All authors analyzed the data and revised the manuscript.

Funding Funding for open access charge: CRUE-Universitat Politècnica de València. This work was supported by Grant PID2020-115763RB-I00 to JMSS from Spanish MICINN. RMM is recipient of a postdoctoral contract (2018/023) from the CDEIGENT program of Generalitat Valenciana. ACS is recipient of a predoctoral contract (FPU17/00715) from the Spanish Ministerio de Educación.
Data availability The data supporting the findings of this study are available within the article.

Code availability Not applicable.

\section{Declarations}

Conflict of interest The authors declare no conflict of interest.

Open Access This article is licensed under a Creative Commons Attribution 4.0 International License, which permits use, sharing, adaptation, distribution and reproduction in any medium or format, as long as you give appropriate credit to the original author(s) and the source, provide a link to the Creative Commons licence, and indicate if changes were made. The images or other third party material in this article are included in the article's Creative Commons licence, unless indicated otherwise in a credit line to the material. If material is not included in the article's Creative Commons licence and your intended use is not permitted by statutory regulation or exceeds the permitted use, you will need to obtain permission directly from the copyright holder. To view a copy of this licence, visit http://creativecommons.org/licenses/by/4.0/.

\section{References}

Ayil-Gutiérrez B, Galaz-Ávalos RM, Peña-Cabrera E, Loyola-Vargas VM (2013) Dynamics of the concentration of IAA and some of its conjugates during the induction of somatic embryogenesis in Coffea canephora. Plant Signal Behav 8(11):e26998

Calabuig-Serna A, Porcel R, Corral-Martínez P, Seguí-Simarro JM (2021) Anther and isolated microspore culture in eggplant (Solanum melongena L.). In: Seguí-Simarro JM (ed) Doubled haploid technology, vol 2: hot topics, apiaceae, brassicaceae, solanaceae. Methods in molecular biology, vol 2288, 1st edn. Humana Press, New York, pp 235-250

Camacho-Fernández C, Hervás D, Rivas-Sendra A, Marín MP, SeguíSimarro JM (2018) Comparison of six different methods to calculate cell densities. Plant Methods 14(1):30. https://doi.org/10. 1186/s13007-018-0297-4

Campion B, de Filippo G, Caporali E, Rossi G, Marziani G (1993) Endogenous growth regulator content in suspension cells of different male and female genotypes of asparagus. Plant Cell Tissue Organ Cult 33(3):281-285. https://doi.org/10.1007/bf02319013

Centeno ML, Rodríguez A, Feito I, Fernández B (1996) Relationship between endogenous auxin and cytokinin levels and morphogenic responses in Actinidia deliciosa tissue cultures. Plant Cell Rep 16(1):58-62. https://doi.org/10.1007/bf01275450

Cheng ZJ, Zhao XY, Shao XX, Wang F, Zhou C, Liu YG, Zhang Y, Zhang XS (2014) Abscisic acid regulates early seed development in Arabidopsis by ABI5-mediated transcription of SHORT HYPOCOTYL UNDER BLUE1. Plant Cell 26(3):1053-1068. https://doi.org/10.1105/tpc.113.121566

Corral-Martínez P, Seguí-Simarro JM (2012) Efficient production of callus-derived doubled haploids through isolated microspore culture in eggplant (Solanum melongena L.). Euphytica 187(1):4761. https://doi.org/10.1007/s10681-012-0715-z

Corral-Martínez P, Seguí-Simarro JM (2014) Refining the method for eggplant microspore culture: effect of abscisic acid, epibrassinolide, polyethylene glycol, naphthaleneacetic acid, 6-benzylaminopurine and arabinogalactan proteins. Euphytica 195(3):369382. https://doi.org/10.1007/s10681-013-1001-4 
Corral-Martínez P, Camacho-Fernández C, Mir R, Seguí-Simarro JM (2021) Doubled haploid production in high- and low-response genotypes of rapeseed (Brassica napus) through isolated microspore culture. In: Seguí-Simarro JM (ed) Doubled haploid technology, vol 2: hot topics, apiaceae, brassicaceae, solanaceae. Methods in molecular biology, vol 2288, 1st edn. Humana Press, New York, pp 129-144

Hays DB, Mandel RM, Pharis RP (2001) Hormones in zygotic and microspore embryos of Brassica napus. Plant Growth Regul 35(1):47-58

Jouannic S, Champion A, Seguí-Simarro JM, Salimova E, Picaud A, Tregear J, Testillano P, Risueno MC, Simanis V, Kreis M, Henry $\mathrm{Y}(2001)$ The protein kinases AtMAP3Ke1 and BnMAP3Ke1 are functional homologues of $S$. pombe cdc7p and may be involved in cell division. Plant J 26(6):637-649

Li H, Soriano M, Cordewener J, Muiño JM, Riksen T, Fukuoka H, Angenent GC, Boutilier K (2014) The histone deacetylase inhibitor Trichostatin A promotes totipotency in the male gametophyte. Plant Cell 26(1):195-209. https://doi.org/10.1105/tpc.113.116491

Liu Z-H, Hsiao I-C, Pan Y-W (1996) Effect of naphthaleneacetic acid on endogenous indole-3-acetic acid, peroxidase and auxin oxidase in hypocotyl cuttings of soybean during root formation. Bot Bull Acad Sin 37(4):247-253

Miyoshi K (1996) Callus induction and plantlet formation through culture of isolated microspores of eggplant (Solanum melongena L.). Plant Cell Rep 15(6):391-395

Pal AK, Acharya K, Ahuja PS (2012) Endogenous auxin level is a critical determinant for in vitro adventitious shoot regeneration in potato (Solanum tuberosum L.). J Plant Biochem Biotechnol 21(2):205-212. https://doi.org/10.1007/s13562-011-0092-z

Parra-Vega V, Renau-Morata B, Sifres A, Seguí-Simarro JM (2013) Stress treatments and in vitro culture conditions influence microspore embryogenesis and growth of callus from anther walls of sweet pepper (Capsicum annuиm L.). Plant Cell Tissue Organ Cult 112(3):353-360. https://doi.org/10.1007/s11240-012-0242-6

Pescador R, Kerbauy GB, de Melo FW, Purgatto E, Suzuki RM, Guerra MP (2012) A hormonal misunderstanding in Acca sellowiana embryogenesis: levels of zygotic embryogenesis do not match those of somatic embryogenesis. Plant Growth Regul 68(1):6776. https://doi.org/10.1007/s10725-012-9694-2

Ramesar-Fortner NS, Yeung EC (2006) Physiological influences in the development and function of the shoot apical meristem of microspore-derived embryos of Brassica napus "Topas." Can J Bot 84(3):371-383

Rivas-San Vicente M, Plasencia J (2011) Salicylic acid beyond defence: its role in plant growth and development. J Exp Bot 62(10):3321-3338. https://doi.org/10.1093/jxb/err031

Rivas-Sendra A, Campos-Vega M, Calabuig-Serna A, Seguí-Simarro JM (2017) Development and characterization of an eggplant (Solanum melongena) doubled haploid population and a doubled haploid line with high androgenic response. Euphytica 213(4):89. https://doi.org/10.1007/s10681-017-1879-3

Rivas-Sendra A, Corral-Martínez P, Camacho-Fernández C, Porcel R, Seguí-Simarro JM (2020) Effects of growth conditions of donor plants and in vitro culture environment in the viability and the embryogenic response of microspores of different eggplant genotypes. Euphytica 216(11):167. https://doi.org/10.1007/ s10681-020-02709-4

Salas P, Prohens J, Seguí-Simarro JM (2011) Evaluation of androgenic competence through anther culture in common eggplant and related species. Euphytica 182(2):261-274. https://doi.org/ 10.1007/s10681-011-0490-2

Salas P, Rivas-Sendra A, Prohens J, Seguí-Simarro JM (2012) Influence of the stage for anther excision and heterostyly in embryogenesis induction from eggplant anther cultures. Euphytica 184(2):235-250. https://doi.org/10.1007/s10681-011-0569-9

Satpute G, Long H, Seguí-Simarro JM, Risueño MC, Testillano PS (2005) Cell architecture during gametophytic and embryogenic microspore development in Brassica napus. Acta Physiol Plant 27(4B):665-674

Seguí-Simarro JM (2016) Androgenesis in solanaceae. In: Germanà MA, Lambardi M (eds) In vitro embryogenesis. Methods in molecular biology, vol 1359. Springer, New York, pp 209-244

Seguí-Simarro JM, Corral-Martínez P, Parra-Vega V, GonzálezGarcía B (2011) Androgenesis in recalcitrant solanaceous crops. Plant Cell Rep 30(5):765-778. https://doi.org/10.1007/ s00299-010-0984-8

Seguí-Simarro JM, Jacquier NMA, Widiez T (2021) Overview of in vitro and in vivo doubled haploid technologies. In: SeguíSimarro JM (ed) Doubled haploid technology, vol 1: general topics, alliaceae, cereals. Methods in molecular biology, vol 2287, 1 st edn. Humana Press, New York, pp 3-22

Seo M, Jikumaru Y, Kamiya Y (2011) Profiling of hormones and related metabolites in seed dormancy and germination studies. In: Kermode AR (ed) Seed dormancy: methods and protocols. Humana Press, Totowa, pp 99-111

Touraev A, Heberle-Bors E (2003) Anther and microspore culture in tobacco. In: Maluszynski M, Kasha KJ, Forster BP, Szarejko I (eds) Doubled haploid production in crop plants. Kluwer Academic Publishers, Dordrecht, pp 223-228

van Bergen S, Kottenhagen MJ, van der Meulen RM, Wang M (1999) The role of abscisic acid in induction of androgenesis: a comparative study between Hordeum vulgare L-cvs. Igri and Digger. J Plant Growth Regul 18(3):135-143

Vergne P, Gaillard A (2021) Isolation of staged and viable maize microspores for DH production. In: Seguí-Simarro JM (ed) Doubled haploid technology, vol 1: general topics, alliaceae, cereals. Methods in molecular biology, vol 2287, 1st edn. Humana Press, New York, pp 281-293

Vondráková Z, Eliášová K, Fischerová L, Vágner M (2011) The role of auxins in somatic embryogenesis of Abies alba. Cent Eur J Biol 6(4):587-596. https://doi.org/10.2478/s11535-011-0035-7

Weyen J (2021) Applications of doubled haploids in plant breeding and applied research. In: Seguí-Simarro JM (ed) Doubled haploid technology, vol 1: general topics, alliaceae, cereals. Methods in molecular biology, vol 2287, 1st edn. Humana Press, New York, pp 23-39

Yan SP, Yang RH, Wang F, Sun LN, Song XS (2017) Effect of auxins and associated metabolic changes on cuttings of hybrid aspen. Forests 8(4):117

Żur I, Dubas E, Krzewska M, Janowiak F (2015) Current insights into hormonal regulation of microspore embryogenesis. Front Plant Sci 6:424. https://doi.org/10.3389/fpls.2015.00424

Publisher's Note Springer Nature remains neutral with regard to jurisdictional claims in published maps and institutional affiliations. 